\title{
Thermodynamic Study on the Protonation Reactions of Glyphosate in Aqueous Solution: Potentiometry, Calorimetry and NMR spectroscopy
}

Bijun Liu ${ }^{\dagger}$, Lan Dong ${ }^{\dagger}$, Qianhong $\mathrm{Yu}^{\dagger}$, Xingliang $\mathrm{Li}^{*}{ }^{* \dagger}$, Fengchang $\mathrm{Wu}^{*}{ }^{* \dagger}$, Zhaoyi Tan ${ }^{\dagger}$, and Shunzhong Luo ${ }^{*}+\dagger$

${ }^{\dagger}$, Institute of Nuclear Physics and Chemistry, China Academy of Engineering Physics, Mianyang, Sichuan 621999, China

‡, State Key Laboratory of Environmental Criteria and Risk Assessment, Chinese Research Academy of Environmental Sciences, Beijing 100012, China

Address correspondence to

Xingliang Li, E-mail: xingliang@caep.cn

Fengchang Wu, E-mail: wufengchang@ vip.skleg.cn

Shunzhong Luo, E-mail: luoshzh@caep.cn 
Table S1 Calorimetric Titration Data for Protonation of Glyphosate Studies

\begin{tabular}{ccccc}
\hline Titration \# & $n_{\mathrm{L}}^{\mathrm{o}}, \mu \mathrm{mol}$ & $n_{\mathrm{H}^{+}}^{\mathrm{o}}, \mu \mathrm{mol}$ & $V^{\mathrm{o}}, \mu \mathrm{L}$ & $C_{\mathrm{NaOH}, \text { titrant }} \mathrm{mM}$ \\
1 & 6.676 & 14.027 & 750 & 0.0577 \\
2 & 4.214 & 17.164 & 750 & 0.0577 \\
3 & 5.572 & 16.863 & 750 & 0.0577 \\
dilution & $1 \mathrm{~mol} \cdot \mathrm{L}^{-1} \mathrm{NaClO}_{4}$ & 750 & 0.0577 \\
\hline
\end{tabular}


Table S2 $Q_{\mathrm{r}, \mathrm{j}}$ Data for the Each Calorimetric Titration

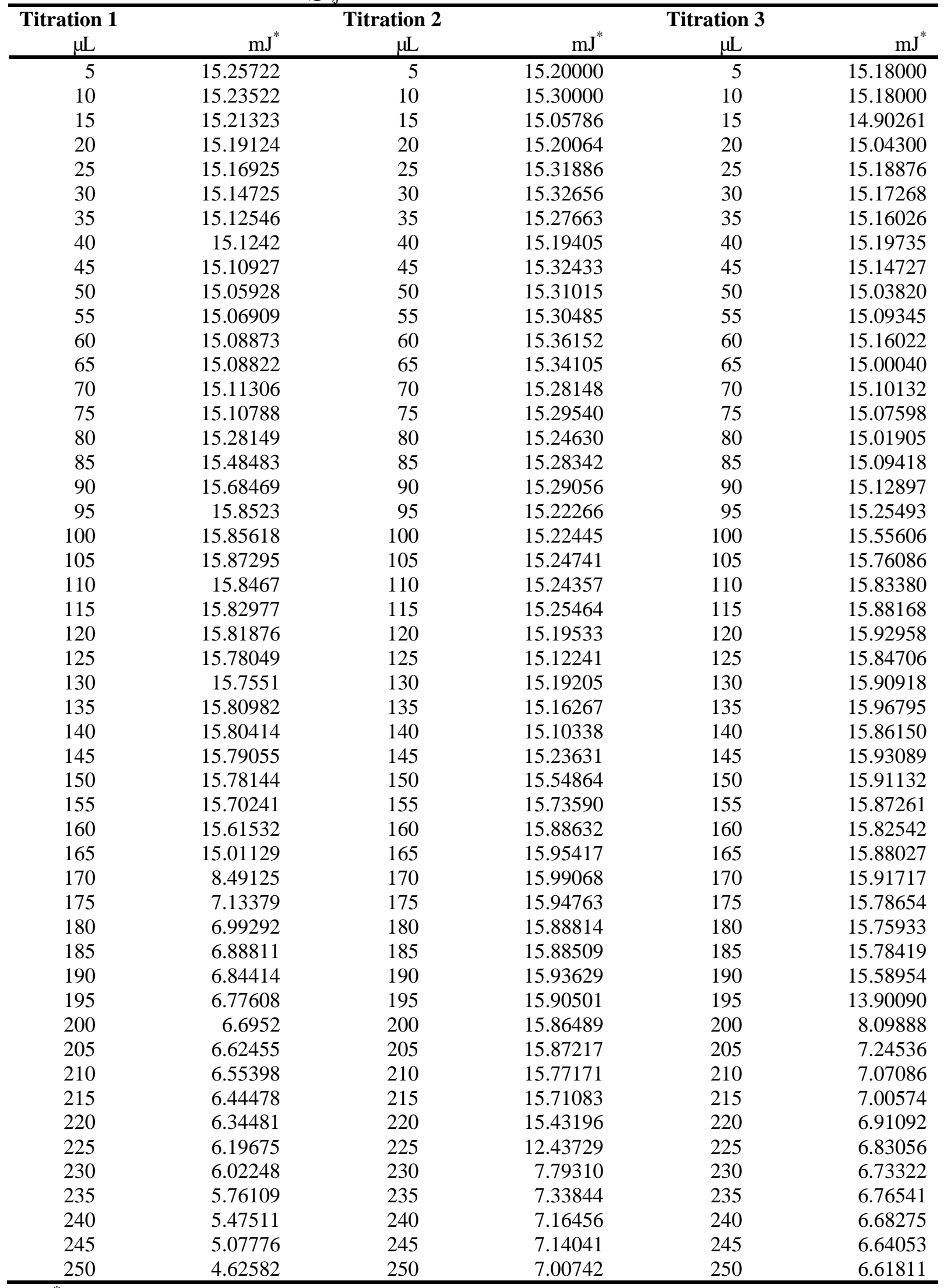

These values were corrected for the heat of dilution of the titrant $\left(Q_{\mathrm{dil}, j}\right)$, which was determined in separate runs $\left(750 \mu \mathrm{L} 1 \mathrm{~mol} \cdot \mathrm{L}^{-1} \mathrm{NaClO}_{4}\right.$ is titrated by $250 \mu \mathrm{L} 0.0577 \mathrm{~mol} \cdot \mathrm{L}^{-1} \mathrm{NaOH}$. Fifty 
injections of $5 \mu \mathrm{L}$ each were performed). The average value of $Q_{\mathrm{dil}, j}$ is $0.021059 \mathrm{~mJ}$ for each injection. 


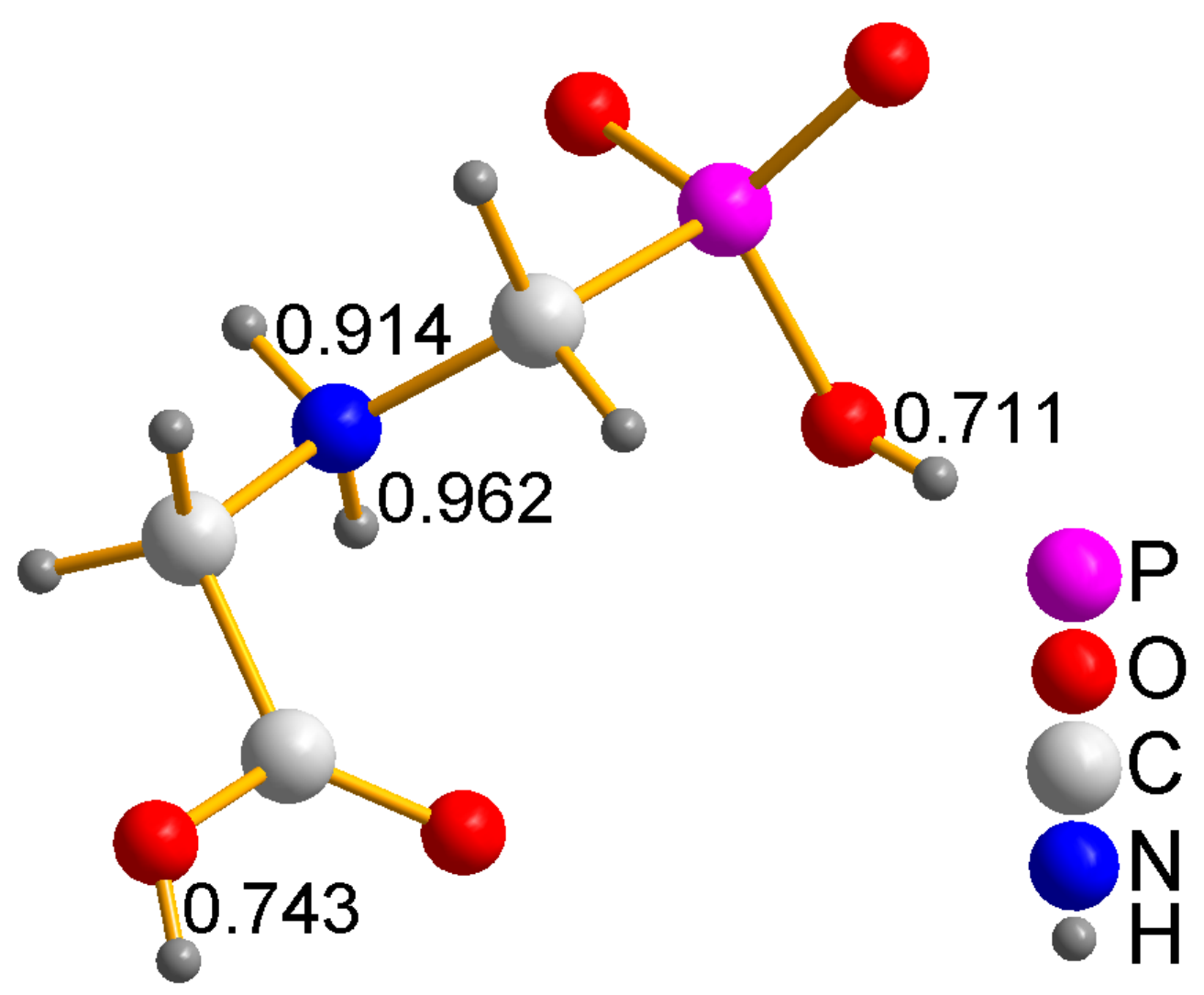

Figure S1 Molecular structure of glyphosate 


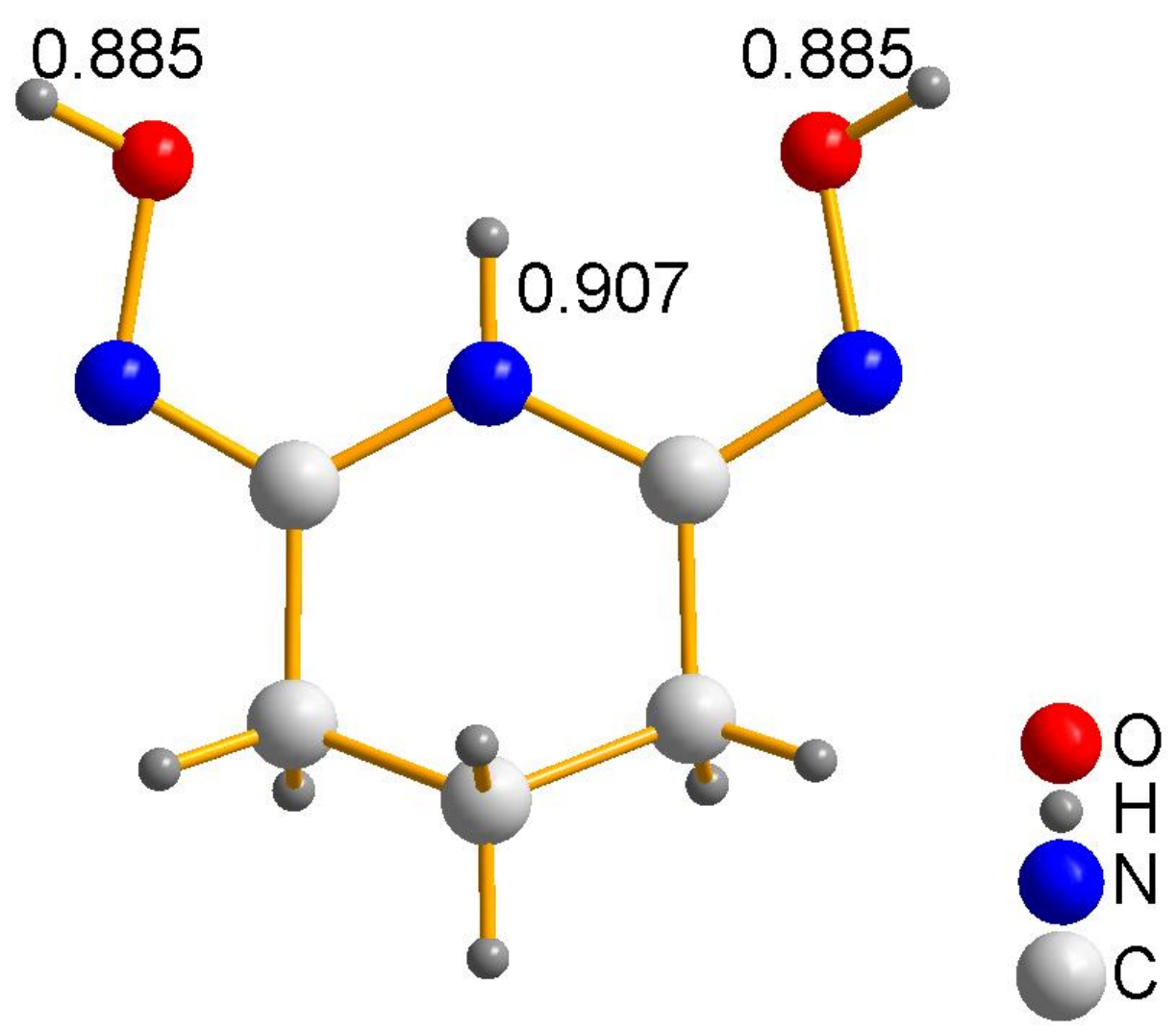

Figure S2 Molecular Structure of Glutaroimide-dioxime 

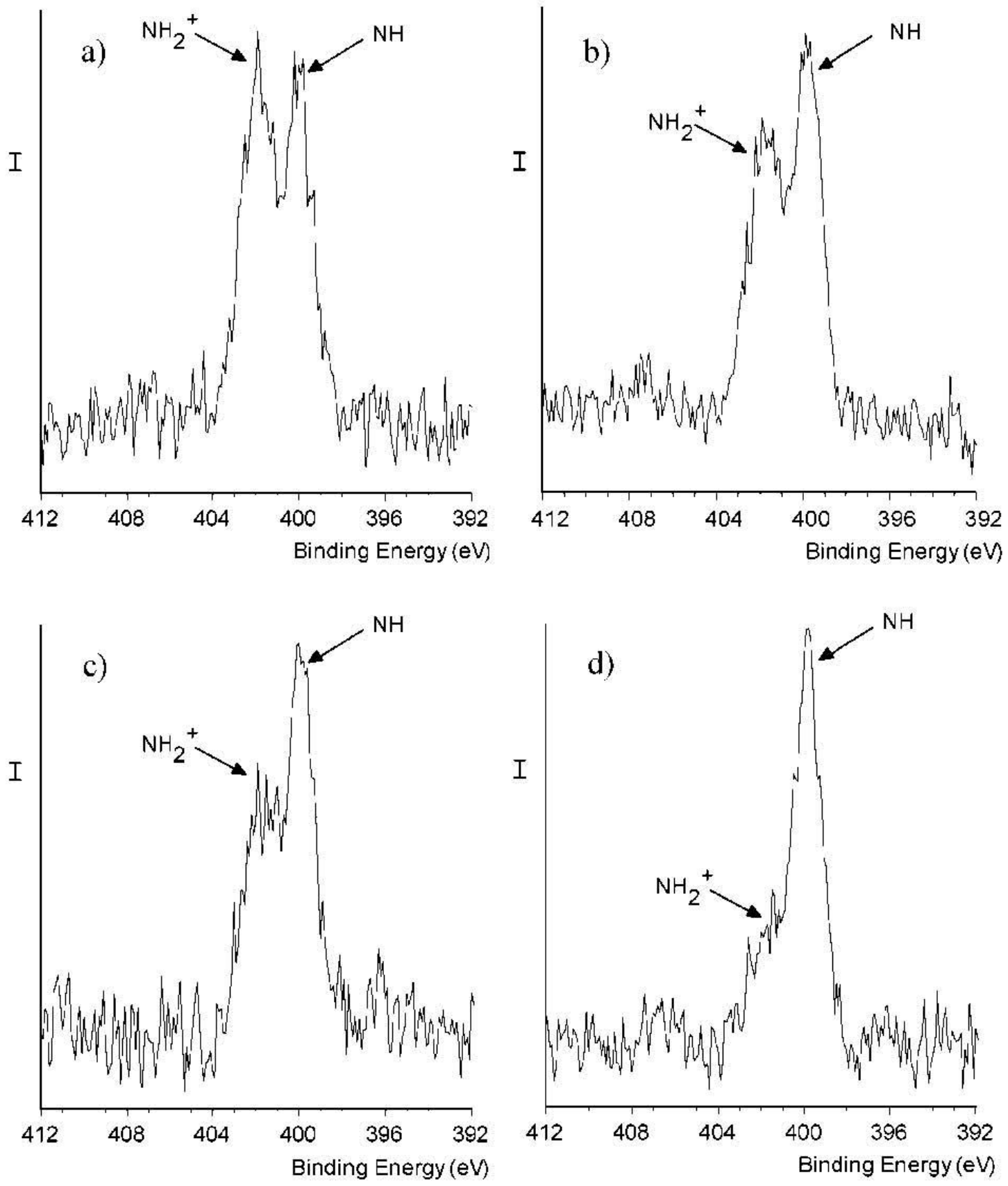

Figure S3 XPS spectra: $\mathrm{pH}$ dependence of N1s: (a) $\mathrm{pH} 3.9$, species $\%: \mathrm{H}_{2} \mathrm{~L}^{-} 94 \%, \mathrm{HL}^{2-}$ 5\%; (b) $\mathrm{pH} 4.9$, species $\%: \mathrm{H}_{2} \mathrm{~L}^{-} 66 \%, \mathrm{HL}^{2-} 33 \%$; (c) $\mathrm{pH} 6.0$, species $\%: \mathrm{H}_{2} \mathrm{~L}^{-} 12 \%$, $\mathrm{HL}^{2-} 87 \%$; (d)pH 8.6, species\%: $\mathrm{HL}^{2-} 90 \%, \mathrm{~L}^{3-} 9 \%$. Species\% in solution calculated by Hyss 2009 programm. $\mathrm{H}_{3} \mathrm{~L}$ stands for the neutral glyphosate. Reprinted with permission from "Julia Sheals, Staffan Sjöberg, Per Persson, Adsorption of Glyphosate on Goethite: Molecular Characterization of Surface Complexes, Environmental Science \& Technology, 2002, 36, 3090-3095". Copyright @ 2002, American Chemical Society 